\title{
Expression of Transforming Growth Factor $\beta 1$ and E-Cadherin Proteins in Pulmonary Adenocarcinoma: Its Significance in Tumor Progression
}

\section{Chi Hong Kim, MD, PhD' \\ Sonya Youngju Park, MD² \\ Jinyoung Yoo, MD, $\mathrm{PhD}^{3}$}

\begin{abstract}
Purpose
This study was conducted in order to investigate the significance of transforming growth factor $\beta 1$ (TGF $\beta 1$ ) and E-cadherin proteins in tumor progression of lung adenocarcinoma and to evaluate their differential expression in association with morphologic characteristics.
\end{abstract}

\section{Materials and Methods}

A total of 65 pulmonary adenocarcinomas were reclassified according to the new classification system proposed by the International Association for the Study of Lung Cancer, American Thoracic Society, and European Respiratory Society. Tumor samples from 20 adenocarcinomas in situ (AIS, formerly bronchioloalveolar carcinoma [BAC]), 9 minimally invasive adenocarcinomas (MIA, formerly BAC with $\leq 5 \mathrm{~mm}$ invasion), 17 lepidic predominant adenocarcinomas (LPA, formerly mixed adenocarcinoma showing nonmucinous BAC features with $>5 \mathrm{~mm}$ invasion), and 19 invasive adenocarcinomas with no BAC features were analyzed by immunohistochemistry for expression of TGFB1 and E-cadherin proteins.

\section{Results}

TGF $\beta 1$ expression was detected in 46\% (21/46) of noninvasive elements and $87 \%$ $(39 / 45)$ of invasive elements $(p=0.001)$. E-Cadherin expression was less frequent in invasive components than in noninvasive components ( $38 \%$ vs. $65 \%, p=0.009$ ). Negative correlation was identified between TGF 1 expression and E-cadherin expression in noninvasive elements $(p=0.022)$. More importantly, significantly higher frequency of TGF $\beta 1$ expression was observed in noninvasive components of LPA (14/17, 82\%), compared with those of either AIS $(5 / 20,25 \%)$ or MIA $(2 / 9,22 \%)(p=0.008)$.

\section{Conclusion}

Our data indicate involvement of both TGF $\beta 1$ and E-cadherin proteins in tumor progression of pulmonary adenocarcinoma. It is noteworthy that TGF $\beta 1$ up-regulation precedes alveolar destruction by invasion of tumor cells. TGFß1 may thus have the potential to improve lung adenocarcinoma diagnostics and therapeutics.

\footnotetext{
Correspondence: Jinyoung
Department of Pathology,

St. Vincent's Hospital,

The Catholic University of Korea

College of Medicine, 93 Jungbu-daero,

Paldal-gu, Suwon 442-723, Korea

Tel: $82-31-249-7593$

Fax: 82-31-244-6786

E-mail: jinyyoo@catholic.ac.kr

Received November 8, 2012

Accepted February 14, 2013
}

\author{
Key words \\ Lung, Adenocarcinoma, \\ Transforming growth factor beta1, Cadherins
}

\section{Introduction}

A bronchioloalveolar carcinoma (BAC) pattern is defined as growth of neoplastic cells along preexisting alveolar structures (lepidic growth) [1]. Although this pattern is a distinctive feature of BAC that is noninvasive adenocarcinoma, invasive adenocarcinomas often contain areas of BAC morphology at the tumor periphery. Numerous data over the past decade have demonstrated the heterogeneity of pulmonary adenocarcinomas in terms of clinical, radiologic, pathologic, and, in particular, molecular aspects $[2,3]$. This brought about the rationale for a change in the World Health Organization (WHO) classification of lung adenocarcinoma, 
culminating in a revision by the International Association for the Study of Lung Cancer, American Thoracic Society, and European Respiratory Society (IASLC/ATS/ERS) [3]. In this new classification, the term BAC has been abandoned, and new terms of adenocarcinoma in situ (AIS), minimally invasive adenocarcinoma (MIA), and lepidic predominant adenocarcinoma (LPA) are introduced. The most important issue is invasion. Although molecular and biological events responsible for this acquisition of invasiveness have been discussed in many reports [4-6], they remain to be elucidated.

Transforming growth factor $\beta$ (TGF $\beta$ ), a pleiotropic cytokine comprised of three isoforms in mammalian cells, is involved in a variety of biological processes, including cell proliferation and differentiation, wound healing, embryogenesis, and apoptosis. Function of TGF $\beta$ s as tumor suppressors during early tumorigenesis, but also as mediators of tumor promoting effects in the later stages of cancer, has been demonstrated in clinical and mouse models $[7,8]$. Although several signaling pathways have been implicated in the mechanism of TGF $\beta$ action, precise timing and context by which TGF $\beta$ signaling functions alternatively in suppression of tumor growth or in promotion of tumor cell invasion remain unclear. Findings of a recent investigation in human lung adenocarcinoma cell lines indicated that TGF $\beta 1$ may promote invasion and metastasis via epithelial-to-mesenchymal transition (EMT). E-Cadherin, a cell-surface glycoprotein, is responsible for maintenance of intercellular connection. Loss of E-cadherin expression with subsequent reduction in cell-to-cell adhesion is a hallmark of EMT. Previous studies have reported involvement of TGF $\beta 1$ in regulation of E-cadherin $[9,10]$. However, the relationships between their expression profiles and morphology have not been reported in tumor progression of lung adenocarcinoma.

In the current study, we recategorized lung adenocarcinomas with and without lepidic growth according to the new classification system [3], and analyzed the expression of TGF 1 1 and E-cadherin proteins in each subgroup in order to address the role of TGF $\beta 1$ and E-cadherin in tumor progression of pulmonary adenocarcinoma and to evaluate their differential expression in association with morphologic characteristics.

\section{Materials and Methods}

\section{Patient characteristics}

A total of 65 patients who underwent surgical resection for lung adenocarcinoma at the Catholic University St. Vincent's Hospital from January 1, 2005 to December 31, 2009 were included in this study. All tumors were staged according to the International Union against Cancer (UICC) guidelines of the 6th edition of the TNM classification of malignant tumors. Tumors with lepidic growth were histologically reclassified according to the amount of invasive component, as described recently by the new IASLC/ATS/ERS classification system [3]: 1) AIS ( $\leq 3 \mathrm{~cm}$ formerly BAC as defined by WHO [1] if there was no evidence of invasion; 2) MIA (formerly BAC with focal invasion) if there was a predominant lepidic pattern with an area of invasion comprising $\leq 5 \mathrm{~mm}$ within a tumor mass $\leq 3 \mathrm{~cm}$; 3 ) LPA (formerly nonmucinous BAC pattern with $>5 \mathrm{~mm}$ invasion). We categorized 65 adenocarcinomas into four groups: group 1, 2a, 2b, and 3. A summary of definitions for each group is shown in Table 1. This study protocol was approved by the Institutional Review Board (IRB) of St. Vincent's Hospital at The Catholic University of Korea (IRB no. VC12TIS10190). Informed consent was waived by the IRB. Clinical information was obtained through a computerized database of the tumor registry.

\section{Immunohistochemical analysis}

Whole tissue sections were obtained from 65 formalinfixed, paraffin-embedded tumor specimens, and were

Table 1. Categories of lung adenocarcinoma according to new IASLC/ATS / ERS classification

\begin{aligned} & Group Definitions \\ & Group 1 Adenocarcinoma in situ (formerly nonmucinous BAC) \\ & Group 2 Minimally invasive adenocarcinoma (formerly BAC with invasive area 5 mm or less) \\ & $2 \mathrm{a}$ Lepidic predominant invasive adenocarcinoma (formerly nonmucinous BAC pattern with \\ & $2 \mathrm{~b}$ invasive area larger than $5 \mathrm{~mm}$ ) \\ & Invasive adenocarcinoma without BAC pattern \\ & \hline\end{aligned}

IASLC/ATS/ERS, International Association for the Study of Lung Cancer, American Thoracic Society, and European Respiratory Society; BAC, bronchioloalveolar carcinoma. 
analyzed for TGF $\beta 1$ and E-cadherin using immunohistochemical studies. Briefly, histology sections measuring $4 \mu \mathrm{m}$ in thickness were deparaffinized in xylene, and rehydrated through a series of alcohols and water. Endogenous peroxidase activity was blocked by soaking the slides in $3 \% \mathrm{H}_{2} \mathrm{O}_{2}$ at $45^{\circ} \mathrm{C}$ for 10 minutes. For antigen retrieval, the sections were autoclaved in a citrate buffer $(2.1 \mathrm{~g} / \mathrm{L}, \mathrm{pH}$ 6.0) for 15 minutes. The sections were then treated with a protein blocking reagent before incubation overnight at $4^{\circ} \mathrm{C}$ with primary antibodies at a 1:100 dilution: anti-TGFß1 (Santa Cruz Biotech, Santa Cruz, CA) and anti-E-cadherin (Cell Marque, Rocklin, CA). After extensive washing with Tris buffer, immunohistochemical staining was performed using the UltraVision LP detection system (Thermo Fisher Scientific, Fremont, CA). Diaminobenzidine was used as a chromogen and Meyer's hematoxylin was used as a nuclear counterstain.

Positive and negative controls were run in all series. Positive controls were breast carcinoma tissue for TGF $\beta 1$ and pituitary gland for E-cadherin. For negative controls, the

Table 2. Summary of clinicopathologic characteristics

\begin{tabular}{|cc}
\hline Median age $(\mathrm{yr})$ & No. $(\%)$ \\
$\leq 60$ & 66 \\
$>60$ & $19(41)$ \\
Gender & $46(59)$ \\
Male & \\
Female & $28(43)$ \\
\hline Smoking & $37(57)$ \\
Never & \\
Ever & $44(68)$ \\
\hline T & $21(32)$ \\
T1 & \\
T2 & $28(43)$ \\
T3 & $27(41)$ \\
T4 & $3(5)$ \\
N & $7(11)$ \\
N0 & \\
N1-3 & $46(59)$ \\
M & $19(41)$ \\
M0 & \\
M1 & $63(97)$ \\
Stage & $2(3)$ \\
I & \\
II & \\
III & $17(14)$ \\
Group 1 & $19(26)$ \\
Group 2b & \\
Group 3 & \\
\hline
\end{tabular}

primary antibody was omitted during processing. Immunohistochemical and pathological assessments were performed in a blind fashion by an experienced pathologist (J.Yoo). Expression for TGF $\beta 1$ was evaluated according to the percentage of tumor cells with immunoreactivity in their cytoplasm: + , if $\geq 10 \%$ of tumor cells were stained; -, if no detectable expression or $<10 \%$ of tumor cells were stained [11]. The membrane showed strong E-cadherin staining and the cytoplasm showed weak staining. E-Cadherin expression was considered positive when $>90 \%$ of tumor cells showed membranous staining [12].

\section{Statistical analysis}

Statistical analyses were performed using the SPSS ver. 19.0 (SPSS Inc., Chicago, IL). Correlations between histologic subtypes and immunohistochemical expressions were estimated using the chi-square and Fisher's exact tests. A $p$-value of $<0.05$ was considered statistically significant.

\section{Results}

Clinical and pathologic data are shown in Table 2. Of the patients, 28 were men and 37 were women. The median age at diagnosis was 66 years (range, 33 to 82 years). Twenty-one patients were former or current smokers, and 44 were neversmokers. Forty-one patients had stage I disease, six had stage II disease, 16 had stage III disease, and two had stage IV disease. Upon pathology review, 20 tumors were classified into group 1, nine into group 2a, 17 into group $2 \mathrm{~b}$, and 19 into group 3. Of a total of 65 lesions, noninvasive elements were observed in 46 specimens, and invasive elements were observed in 45 (Table 3 ).

Negative and positive controls showed the expected results. More frequent expression of TGF $\beta 1$ was observed in invasive foci than in BAC component ( $87 \%$ vs. $46 \%$ ), and the difference was statistically significant $(\mathrm{p}=0.001)$ (Table 3 , Fig. $1 \mathrm{~A}-\mathrm{D})$. In contrast, the prevalence of E-cadherin expression was significantly high in areas with noninvasive component when compared with that in invasive foci $(65 \%$ vs. $38 \%$, $\mathrm{p}=0.009$ ) (Fig. 1E-H). Expression of TGF $\beta 1$ and E-cadherin showed significant correlation in noninvasive components $(p=0.022)$, but not in invasive foci $(p=0.385)$ (Table 4). In order to further characterize different subgroups of lung adenocarcinoma with and without lepidic growth, we examined the expression of TGF $\beta 1$ and E-cadherin proteins in the noninvasive component and invasive component for each group, respectively. More frequent expression of TGF $\beta 1$ was 

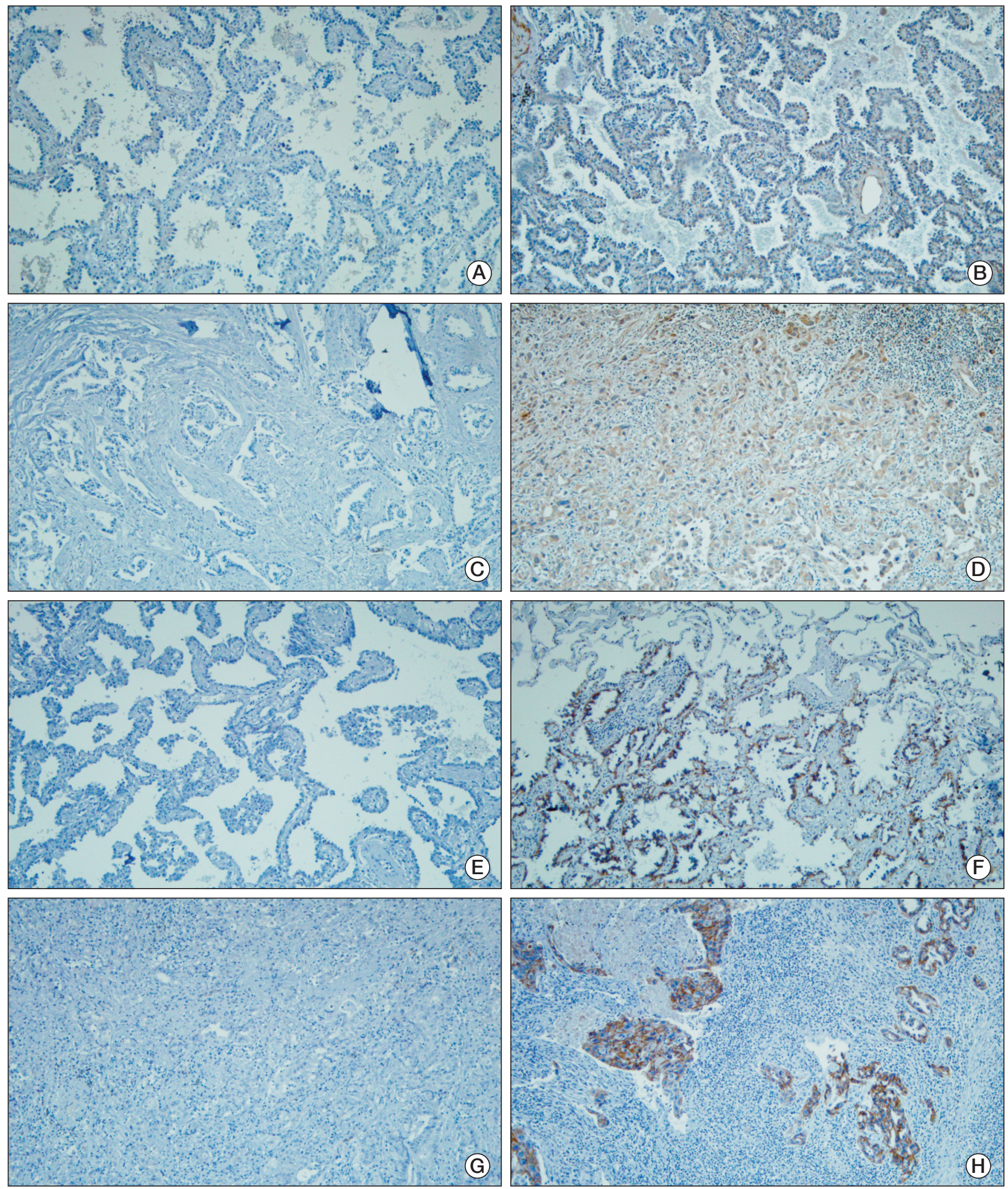

Fig. 1. Representative immunostaining results for transforming growth factor $\beta 1$ (TGFß1) (A-D) and E-cadherin (E-H). Noninvasive component that showed negative (A) and positive (B) staining for TGF $\beta 1$. Invasive component that was negative (C) and up-regulated (D) for TGFß1. Noninvasive element that showed lack of E-cadherin expression (E) and membranous staining $(F)$. Invasive element that was negative $(G)$ and positive $(H)$ for E-cadherin expression $(A-H, A B C$ method, $\times 100)$. 
Table 3. Expression of TGF $\beta 1$ and E-cadherin proteins in each component

\begin{tabular}{lcccc} 
& Noninvasive component & Invasive component & Total & p-value \\
\hline TGF $\beta 1$ & & & & 0.001 \\
Negative & 25 & 6 & 31 & \\
Positive & 21 & 39 & 60 & \\
Positive lesions (\%) & 46 & 87 & - & 0.009 \\
E-cadherin & & & 44 & \\
Negative & 16 & 28 & 47 \\
Positive & 30 & 17 & - & \\
Positive lesions (\%) & 65 & 38 & 91 & \\
Total & 46 & 45 & & \\
\hline
\end{tabular}

TGF $\beta 1$, transforming growth factor $\beta 1$.

Table 4. Correlation between TGF $\beta 1$ expression and E-cadherin expression

\begin{tabular}{|c|c|c|c|c|c|c|}
\hline & \multicolumn{6}{|c|}{ E-cadherin expression } \\
\hline & \multicolumn{3}{|c|}{ Noninvasive component } & \multicolumn{3}{|c|}{ Invasive component } \\
\hline & Negative & Positive & p-value & Negative & Positive & p-value \\
\hline \multicolumn{7}{|c|}{ TGF $\beta 1$ expression } \\
\hline Negative & 5 & 20 & 0.022 & 5 & 1 & 0.385 \\
\hline Positive & 11 & 10 & & 23 & 16 & \\
\hline Total & 16 & 30 & & 28 & 17 & \\
\hline
\end{tabular}

TGF $\beta 1$, transforming growth factor $\beta 1$.

Table 5. Expression of TGF $\beta 1$ and E-cadherin proteins in each component by subgroup

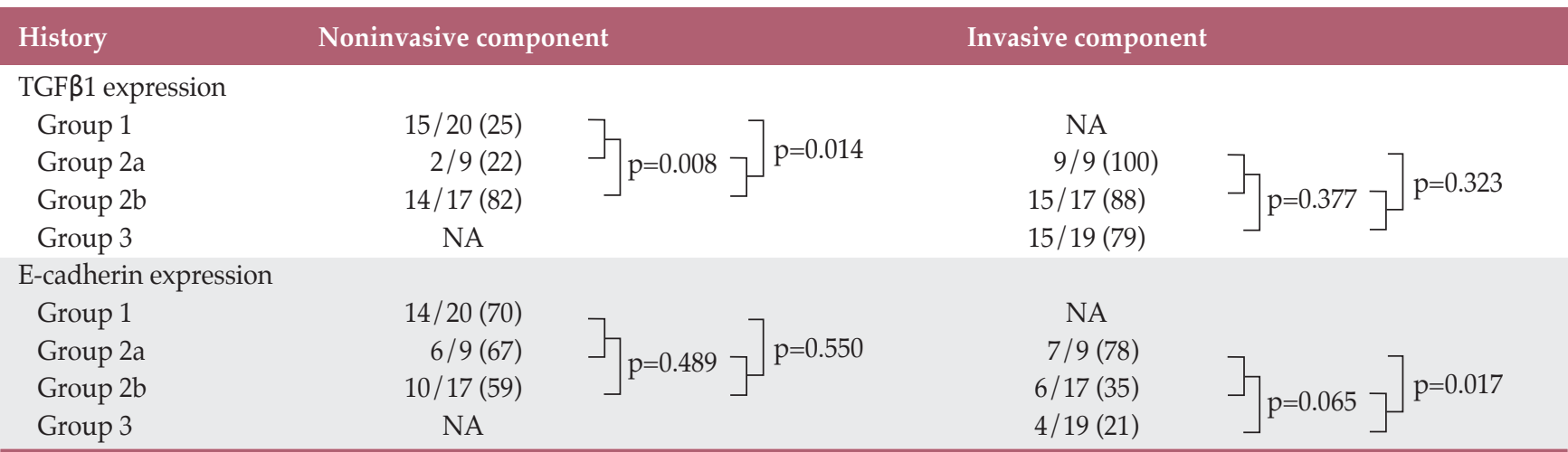

Values are presented as number $(\%)$. TGF $\beta 1$, transforming growth factor $\beta 1$; NA, not available.

observed in the noninvasive component of group $2 \mathrm{~b}(14 / 17$, $82 \%)$ than in that of either group $1(5 / 20,25 \%)$ or group $2 a$ $(2 / 9,22 \%)$ (Table 5$)$. The differences were statistically significant: group 1 vs. group $2 \mathrm{a}+$ group $2 \mathrm{~b}(\mathrm{p}=0.014)$, and group $1+$ group $2 \mathrm{a}$ vs. group $2 \mathrm{~b}(\mathrm{p}=0.008)$. TGF $\beta 1$ expression in invasive foci was demonstrated in $100 \%$ of group $2 a(9 / 9)$, $88 \%$ of group $2 \mathrm{~b}(15 / 17)$, and $79 \%$ of group $3(15 / 19)$; no significant correlations were observed between the following groups: group $2 \mathrm{a}$ vs. group $2 \mathrm{~b}+$ group $3(\mathrm{p}=0.323)$, and group $2 \mathrm{a}+$ group $2 \mathrm{~b}$ vs. group $3(\mathrm{p}=0.377)$. However, in group $2 \mathrm{a}$, expression of TGF $\beta 1$ was observed in all invasive elements, but in two of nine noninvasive elements $(p=0.002)$. Immunoreactivity with anti-E-cadherin in noninvasive elements was exhibited in $70 \%$ (14/20) of group 1, 67\% (6/9) of group 
$2 a$, and $59 \%(10 / 17)$ of group $2 b$. The frequency was lower in group $2 \mathrm{~b}$ than in group $1+$ group $2 \mathrm{a}$, however, it did not reach statistical significance $(\mathrm{p}=0.489)$. No association was found between group 1 and group $2 a+$ group $2 b(p=0.550)$. E-cadherin expression in invasive foci was observed in $78 \%$ (7/9) of group 2a, 35\% (6/17) of group $2 b$, and $21 \%(4 / 19)$ of group 3. Significantly higher frequency was observed in group 2a than in group $2 b+$ group $3(p=0.017)$. The difference in E-cadherin expression between group $2 a+$ group $2 b$ and group 3 approached statistical significance $(\mathrm{p}=0.065)$.

\section{Discussion}

To date, little is known about the sequence of molecular events and morphologic changes in development of invasive lung adenocarcinoma. In particular, it has not yet been determined which of the two precedes the other in stepwise progression. We compared the expression status of TGF $\beta 1$ and E-cadherin proteins between the noninvasiveand invasive components of pulmonary adenocarcinomas with varying degrees of invasion. The major findings of our study are as follows: 1) the frequency of TGF $\beta 1$ overexpression was significantly increased in invasive foci, whereas E-cadherin expression was more common in noninvasive elements; 2 ) TGF $\beta 1$ expression showed inverse correlation with E-cadherin expression in noninvasive components; 3 ) up-regulation of TGF $\beta 1$ occurs not only before loss of E-cadherin but also before the structural destruction of the alveolar wall by invasive tumor cells.

Overexpression of TGF $\beta 1$ has been reported in lung cancer, particularly in association with tumor progression. Hasegawa et al. [13] reported a negative correlation of TGF $\beta 1$ with patient survival in non-small cell lung carcinoma (NSCLC). In their study, TGF $\beta 1$ protein levels were measured in surgically resected NSCLC tissues using enzymelinked immunosorbent assay, and showed correlation with tumor angiogenesis. Other researchers have demonstrated by immunohistochemistry the significance of TGF $\beta 1$ overexpression in metastasis and prognosis of pulmonary adenocarcinoma, however, they did not divide the tumors into subgroups or mention whether BACs were included in the samples [14,15].

Molecular studies in different subtypes of lung adenocarcinoma are rather limited. In a study of small early lung adenocarcinoma reported by Aoyagi et al. [6], a significant increase in the incidence of loss of heterozygosity $(\mathrm{LOH})$ was observed with a more invasive morphology: $16.7 \%$ of Noguchi type A tumors (localized bronchioloalvelar carcinoma [LBAC]) vs. $39.3 \%$ of Noguchi type B tumors (LBAC with alveolar collapse) vs. $96.2 \%$ of Noguchi type C tumors (LBAC with active fibroblastic proliferation). In addition, in type $\mathrm{C}$ tumors, $\mathrm{LOH}$ was observed more frequently in central fibrotic areas than in peripheral BAC areas. This finding suggests that tumor cells in fibrotic regions are molecularly different from those in peripheral BAC regions, and are more genetically altered and malignant. Using DNA microarray analysis, Borczuk et al. [16], who examined gene expression signatures associated with invasiveness in three subgroups of pulmonary adenocarcinoma (BAC, mixed adenocarcinoma with BAC, and invasive carcinoma), reported that the gene expression signature of purely invasive tumors was distinct from those of other adenocarcinomas, and the repression of type II TGF $\beta$ receptor may act as a significant determinant of lung adenocarcinoma invasiveness. In the current study, we examined lung adenocarcinomas encompassing a spectrum of invasion using immunohistochemistry to demonstrate TGF $\beta 1$ status in vivo. In agreement with previous reports [13-16], our data showed a high frequency of TGF $\beta 1$ expression in invasive foci compared with noninvasive foci, which supports the involvement of TGF $\beta 1$ upregulation in tumor invasion. However, of LPAs, TGF $\beta 1$ expression showed a significant increase at similar frequencies in both noninvasive and invasive elements $(82 \%$ vs. $88 \%$ ). In addition, in the noninvasive components of each subgroup, a significant difference was observed between LPA and AIS or MIA; expression of TGF $\beta 1$ was much more frequent in LPA than in the other two subgroups. This finding suggests that tumor cells of noninvasive areas of LPA are morphologically similar to but molecularly different from those of AIS and MIA. This observation is important because TGF $\beta 1$ up-regulation appears to occur before morphologic destruction of the alveolar wall by invasion of tumor cells. Consistently high incidence of TGF $\beta 1$ expression in the invasive components of each subgroup further supports TGF $\beta 1$ up-regulation as an early event in the invasive process. Therefore, detection of TGF $\beta 1$ overexpression in small biopsy specimens showing adenocarcinoma with pure lepidic growth may have the potential for assumption of stromal invasion somewhere within the tumor and may be of great value in discriminating an AIS from a lepidic pattern of growth within an otherwise invasive adenocarcinoma. Western blotting of micro-dissected tumor samples from each component of each subgroup is currently in progress in our laboratory for validation.

Negative correlation between E-cadherin expression and invasiveness has been well documented in various cancers. In keeping with previous data [12,17], findings of our study demonstrated that loss of E-cadherin was increased as lung adenocarcinoma underwent histologic progression. E-Cadherin expression showed inverse correlation with TGF $\beta 1$ expression. In addition, no significant change in E-cadherin 
expression, but a marked increase in the rate of TGF $\beta 1$ expression, was detected in the noninvasive elements of LPA. These findings provide morphologic evidence that loss of E-cadherin occurs after TGF $\beta 1$ up-regulation, and that TGF $\beta 1$ down-regulates E-cadherin. Studies demonstrating involvement of TGF $\beta 1$ in down-regulation of E-cadherin, various signaling pathways of which have been implicated for possible mechanisms, have been reported $[9,10,18]$. Findings from a recent investigation in lung cancer cells demonstrated regulation of TGF $\beta$-induced EMT by miR-23a through suppression of E-cadherin [9]. Further work on the biological control mechanism through EMT is needed in pulmonary adenocarcinoma subclasses in order to attain a better understanding of the sequential alterations of TGF $\beta 1$ and E-cadherin that may have morphological associations.

\section{Conclusion}

The current study demonstrated significant differences in TGF $\beta 1$ and E-cadherin expression between the noninvasive and invasive components of lung adenocarcinoma, indicating involvement of both TGF $\beta 1$ and E-cadherin proteins in tumor progression. Of noninvasive elements of each group, LPA exhibited TGF $\beta 1$ expression more frequently than AIS or MIA. In addition, despite being morphologically noninvasive, TGF $\beta 1$ expression was detected in noninvasive areas of LPA as compared to the incidence observed in invasive regions of each group. These findings suggest that TGF $\beta 1$ up-regulation is an early event in the invasive process, preceding structural destruction of the alveolar wall by invasion of tumor cells. Conduct of additional large-scale studies is warranted; the results of such investigation may be helpful in assessment of invasiveness in small biopsy specimens of pure lepidic growth.

\section{Conflicts of Interest}

Conflict of interest relevant to this article was not reported.

\section{References}

1. Travis WD, Brambilla E, Muller-Hermelink HK, Harris CC. World Health Organization classification of tumours: pathology and genetics of tumours of the lung, pleura, thymus and heart. Lyon: IARC Press; 2004.

2. Yoshizawa A, Motoi N, Riely GJ, Sima CS, Gerald WL, Kris MG, et al. Impact of proposed IASLC/ ATS/ERS classification of lung adenocarcinoma: prognostic subgroups and implications for further revision of staging based on analysis of 514 stage I cases. Mod Pathol. 2011;24:653-64.

3. Travis WD, Brambilla E, Noguchi M, Nicholson AG, Geisinger KR, Yatabe Y, et al. International Association for the Study of Lung Cancer/American Thoracic Society/European Respiratory Society international multidisciplinary classification of lung adenocarcinoma. J Thorac Oncol. 2011;6:244-85.

4. Soh J, Toyooka S, Ichihara S, Asano H, Kobayashi N, Suehisa $\mathrm{H}$, et al. Sequential molecular changes during multistage pathogenesis of small peripheral adenocarcinomas of the lung. J Thorac Oncol. 2008;3:340-7.

5. Aviel-Ronen S, Coe BP, Lau SK, da Cunha Santos G, Zhu CQ, Strumpf D, et al. Genomic markers for malignant progression in pulmonary adenocarcinoma with bronchioloalveolar features. Proc Natl Acad Sci U S A. 2008;105:10155-60.

6. Aoyagi Y, Yokose T, Minami Y, Ochiai A, Iijima T, Morishita $Y$, et al. Accumulation of losses of heterozygosity and multistep carcinogenesis in pulmonary adenocarcinoma. Cancer Res. 2001;61:7950-4.

7. Derynck R, Akhurst RJ, Balmain A. TGF-beta signaling in tumor suppression and cancer progression. Nat Genet. 2001; 29:117-29.

8. Roberts AB, Wakefield LM. The two faces of transforming growth factor beta in carcinogenesis. Proc Natl Acad Sci U S A. 2003;100:8621-3.

9. Cao M, Seike M, Soeno C, Mizutani H, Kitamura K, Minegishi $\mathrm{Y}$, et al. MiR-23a regulates TGF-beta-induced epithelialmesenchymal transition by targeting E-cadherin in lung cancer cells. Int J Oncol. 2012;41:869-75.

10. Herfs M, Hubert P, Kholod N, Caberg JH, Gilles C, Berx G, et al. Transforming growth factor-beta1-mediated Slug and Snail transcription factor up-regulation reduces the density of Langerhans cells in epithelial metaplasia by affecting Ecadherin expression. Am J Pathol. 2008;172:1391-402.

11. Saito H, Tsujitani S, Oka S, Kondo A, Ikeguchi M, Maeta M, et al. The expression of transforming growth factor-beta1 is significantly correlated with the expression of vascular endothelial growth factor and poor prognosis of patients with advanced gastric carcinoma. Cancer. 1999;86:1455-62.

12. Awaya H, Takeshima Y, Amatya VJ, Ishida H, Yamasaki M, Kohno N, et al. Loss of expression of E-cadherin and betacatenin is associated with progression of pulmonary adenocarcinoma. Pathol Int. 2005;55:14-8.

13. Hasegawa Y, Takanashi S, Kanehira Y, Tsushima T, Imai T, Okumura K. Transforming growth factor-beta1 level correlates with angiogenesis, tumor progression, and prognosis in patients with nonsmall cell lung carcinoma. Cancer. 
2001;91:964-71.

14. Takanami I, Imamura T, Hashizume T, Kikuchi K, Yamamoto Y, Kodaira S. Transforming growth factor beta 1 as a prognostic factor in pulmonary adenocarcinoma. J Clin Pathol. 1994;4 7:1098-100.

15. Saji H, Nakamura H, Awut I, Kawasaki N, Hagiwara M, Ogata A, et al. Significance of expression of TGF-beta in pulmonary metastasis in non-small cell lung cancer tissues. Ann Thorac Cardiovasc Surg. 2003;9:295-300.

16. Borczuk AC, Kim HK, Yegen HA, Friedman RA, Powell CA. Lung adenocarcinoma global profiling identifies type II trans- forming growth factor-beta receptor as a repressor of invasiveness. Am J Respir Crit Care Med. 2005;172:729-37.

17. Kalogeraki A, Bouros D, Zoras O, Karabekios S, Chalkiadakis G, Stathopoulos E, et al. E-cadherin expression on fine-needle aspiration biopsies in primary lung adenocarcinomas is related to tumor differentiation and invasion. Anticancer Res. 2003;23:3367-71.

18. Zhang HJ, Wang HY, Zhang HT, Su JM, Zhu J, Wang HB, et al. Transforming growth factor-beta1 promotes lung adenocarcinoma invasion and metastasis by epithelial-to-mesenchymal transition. Mol Cell Biochem. 2011;355:309-14. 